\title{
Designing a pilot model of compressorless combined cycle unit
}

\author{
Vladimir Volkov-Muzylev ${ }^{1, *}$, Yuri Borisov ${ }^{1,2}$, and Nikolay Fominykh ${ }^{1}$ \\ ${ }^{1}$ JIHT RAS, 125412 Izhorskaya Str., Bldg. 13, Block 2, Moscow, Russia \\ ${ }^{2}$ BMSTU, 105005 2nd Baumanskaya Str., Bldg. 5, Block 1, Moscow, Russia
}

\begin{abstract}
The article is dedicated to a design of a pilot model of a $60 \mathrm{MW}$ installation, operating according to a new promising compressorless combined cycle. In the course of the study, the weight-size parameters and functional indicators of the main parts of the energy complex were preliminarily determined. The auxiliary components of a compressorless combined cycle unit were selected, such as pumps, heat exchangers, compressors, pipelines and etc. Within a draft elaboration, preliminary weight, size and functional parameters of a turbine unit were defined, solutions were found for two groups of pumping equipment, and a plant prototype was designed. Additionally, the weight and size parameters of the plate heat exchangers were determined and a special design of shell-and-tube heat exchangers was designed to return the heat of combustion products. The results, obtained during the study, make it possible to simulate the behavior (develop dynamic models) of a compressorless combined cycle unit in all operating modes (from start-up to shutdown) under various conditions, as well as to carry out a feasibility study for the creation of such installations.
\end{abstract}

\section{Introduction}

Currently, power industry occupies an important place in activities of the human being. Consequently, humanity is constantly striving to improve the existing technologies for the production of electric and thermal energy, as well as to develop new schemes and cycles, that meet the modern economic and environmental requirements. Thus, in addition to efficient energy production, it is necessary to pay attention to the impact of the created installations on the environment, especially the emissions of carbon dioxide into the atmosphere. In works [1-6], a cycle of a compressorless combined cycle unit (CCCU) is considered, which main distinct feature is its focus on the combined production of heat and electric energy. At the same time, CCCU allows to independently regulate the parameters, affecting the ratio of generated heat and electric power, temperature at the turbine inlet, and also permits to separate $\mathrm{CO}_{2}$ from combustion products in a liquid form, being convenient for further utilization.

This research is devoted to the pilot design of the 60 MW compressorless combined cycle unit. The purpose of the work was to preliminary determine the weight, size and functional indicators of the main parts of the power complex with the capacity of $60 \mathrm{MW}$, operating according to CCCU cycle. This will allow to develop dynamic models of CCCU in all modes of operation from start-up to shutdown under various conditions, as

\footnotetext{
${ }^{*}$ Corresponding author: vladimir_muzylev@mail.ru
}

well as to carry out the feasibility study for the creation of such installations.

\section{Designing of $\mathrm{CCCU}$}

Before designing $\mathrm{CCCU}$, the analysis of a schematic diagram, described in the article [1], was carried out. Based on the thermodynamic calculation of CCCU, the main parameters of equipment, involved in the operation of the cycle and necessary to ensure its uninterrupted operation, were obtained (fuel, oxygen, carbon dioxide and water pumps, heat exchangers, turbine unit, auxiliary carbon dioxide compressor).

Thus, a search for equipment with the necessary characteristics for the operation of CCCU in the nominal mode was carried out. At the initial stage of work, equipment was divided into two groups: main and auxiliary. The main equipment includes elements of the installation, that need to be designed for specific parameters of the combined cycle plant, for example, a combustion chamber, a turbine unit, and electric generators. Equipment, the varieties of which exist on the market, is assigned to the auxiliary group. It includes pumps, heat exchangers, compressors, pipelines, etc.

The selection has begun with pumps and their electric motors. Based on thermodynamic calculations, pumping equipment can be separated into two groups: high-pressure and high-flow. Plunger pumps (for example, manufactured by Uraca) were selected as devices for creating a high pressure (from 3 to 10 thousand meters of the pumped medium column). It is proposed to provide a high flow rate of operating fluid 
(over $500 \mathrm{~kg} / \mathrm{s}$ ) using the horizontal centrifugal pumps (for example, manufactured by KSB). After that, asynchronous explosion-proof electric motors with a power reserve of $10-15 \%$ were selected.

The pressure-flow characteristic of plunger pumps is "steep". The flow rate is practically independent of the pressure generated by the pump at a constant speed of rotation. In addition, the maximum possible pump head is practically independent of the speed. If it is necessary to regulate the flow rate of the working medium through such a pump, it is most reasonable to change the rotational speed (most economically).

In contrast to positive-displacement piston machines, the pressure-flow characteristic of centrifugal pumps has a "flat" character. At a constant speed, the flow rate is very much dependent on the pressure generated by the pump. Also, the maximum possible head created by the pump is significantly influenced by the rotational speed of the impellers. Therefore, it is problematic to regulate pumps of this class by changing the speed. The most common and economical way (however, less technologically advanced) to control centrifugal pumps is the inlet blades turning.

To implement heat transfer processes in the cycle, Alfa Laval plate heat exchangers were proposed. This type of heat exchangers was chosen because of a number of significant advantages over shell-and-tube ones. The heat transfer coefficient of plate heat exchangers is 3-5 times higher than the heat transfer coefficient of shell and tube heat exchangers. In addition to this, plate heat exchangers have significantly smaller weight and size parameters. They are 3-10 times less than that of shelland-tube heat exchangers, depending on their capacity, which is important for the research station. Also, their advantage over shell-and-tube devices is less susceptibility to vibration. Rolling joints of shell-andtube heat exchangers lose their tightness even from slight vibrations. It is fraught with the overflow of one medium into another, depending on the higher pressure. The change in the power of the plate heat exchanger occurs by increasing or decreasing the heat exchange surface. For instance, it is enough to simply change the number of plates in the package, while increasing only one of the overall dimensions. In the case of shell-and-tube heat exchangers, the change in power is much more difficult.

At the first stage, the choice of heat exchangers was carried out using an approximate calculation for heat output. Determination of the amount of heat, removed from hot to cold heat transfer fluid, was determined by the following equation:

$$
Q=k \cdot F \cdot t_{a v}
$$

where $k$ - heat-transfer coefficient, $\mathrm{W} /\left(\mathrm{m}^{2} \cdot \mathrm{K}\right) ; F$ heat exchange surface, $\mathrm{m}^{2} ; t_{a v}$ - average temperature head, $\mathrm{K}$.

For initial estimates of heat exchange equipment, the calculation was carried out according to criterion dependencies:

$$
N u=f(\operatorname{Re} ; \operatorname{Pr})
$$

The values of heat exchange surface were taken from the data of supply catalogs.

After the completion of construction, during the debugging phase of the CCCU cycle work, it is planned to bring and pump liquefied methane and $\mathrm{O}_{2}$ into vertical tanks, which are special cryogenic containers. When all processes are established and the station delivers the rated power, it is possible to build an air separation unit or connect $\mathrm{CCCU}$ to a liquefied gas plant. For instance, if it is available in nearby areas.

In the process of creating the CCCU cycle, it has managed to get rid of most of the compressor equipment, replacing it with pumps. However, it is necessary to install a compressor station between the low and high pressure contact condensers (to prevent water freezing). It allows to increase the pressure of gaseous $\mathrm{CO}_{2}$ to the required value depending on the changing conditions at the suction. At this stage, an Atlas Copco compressor unit, based on a screw compressor, has been selected.

The cold of LNG and $\mathrm{O}_{2}$ are used to liquefy carbon dioxide, as well as an additional refrigeration unit, which is able to remove $30 \mathrm{MW}$ of heat from the $\mathrm{CO}_{2}$ liquefaction device. Bitzer screw blocks with a cooling capacity of $1400 \mathrm{~kW}$ were selected as refrigeration compressors (Fig. 1).
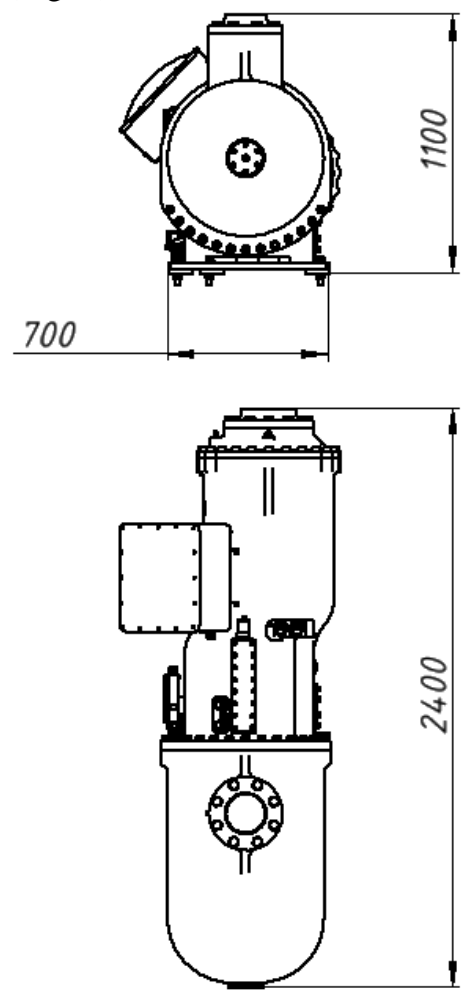

Fig. 1. Overall dimensions of the refrigerating screw block.

The compressed refrigerant is liquefied in a Refrion Ecooler, which is closed cooling tower. The design of such unit is a standard closed cooling circuit with a coolant and irrigation panels with a water recirculation system, equipped with fans (Fig. 2). 


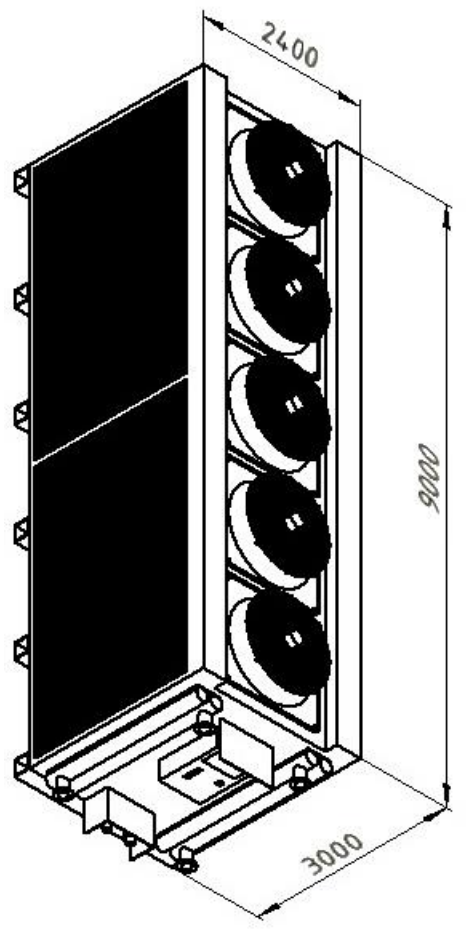

Fig. 2. Overall dimensions of the closed cooling tower.

The adiabatic panels, located between the heat exchangers and the outer protective grid, are uniformly wetted through the water recovery distribution system. The air, passing through the panels, is humidified and cooled with values that change depending on the operating conditions. Water consumption is limited only by the evaporated water. This leads to water savings up to $90 \%$ compared to the consumption of equipment such as evaporation towers. This cooling tower has the following features:

1) automatic drainage system that prevents water stagnation, all water is automatically drained every 24 hours;

2) the system prevents the spread of Legionella by preventing droplets from entering the air stream;

3) ease of maintenance due to full access to the inside of the unit;

4) all components of the hydraulic system are accessible for inspection.

In addition, it is proposed the design of shell-andtube heat exchangers for returning the heat of combustion products to LNG and liquid oxygen. The heat exchange surface is formed by long curved tubes, bent along an involute profile so as to uniformly fill the space between two cylindrical surfaces. Thus, such a heat exchanger can be easily mounted in a pipeline, used for removing combustion products after expansion in the turbine block (Fig. 3).

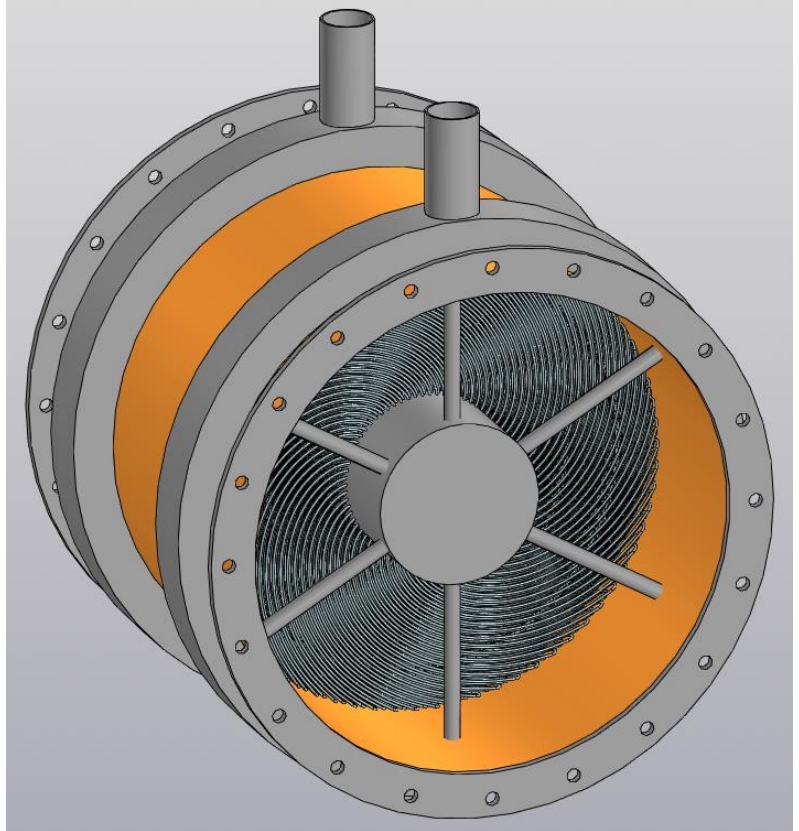

Fig. 3. Shell-and-tube heat exchangers for returning the heat of combustion products to LNG and liquid oxygen.

It should be noted that the geometric parameters of the tubes directly depend only on the diameter and manufacturing technology. The selected tube has an outer diameter of $6 \mathrm{~mm}$ and a wall thickness of $1 \mathrm{~mm}$. On the one hand, it ensures compliance with the strength standards. On the other hand, it minimizes the heat exchanger dimensions (with the required degree of recuperation). At the same time, it is technologically possible to manufacture such a recuperator using modern laser welding technologies. The body of the heat exchanger section has a diameter of 1.2 meters and a length of 0.8 meters. To achieve the required value of the energy recovered in the heat exchanger, the required number of sections can be placed in the pipelines without significantly changing the design of the entire installation as a whole.

Within the framework of the elaboration, preliminary weight, size and functional parameters of the turbine unit were determined. Firstly, high-pressure combustion products expand in central part of unit. After that, it is successively supplied to left and right turbines. Thus, there is a three-stage combustion products expansion process to generate energy by electric generators, connected to the shafts. Each stage is enclosed in a separate housing, the free space of which is filled with pressurized water. There are channels with a certain hydraulic resistance between each section filled with water. Thus, it occurs the pressure decrease when water flows between the sections. This is done to sufficiently decrease the load from the structural elements of each stage. Also, it will greatly simplify and reduce the cost of production. The impellers of each stage are installed by the back side of turbine wheel to each other to relieve the axial load (Fig. 4). 


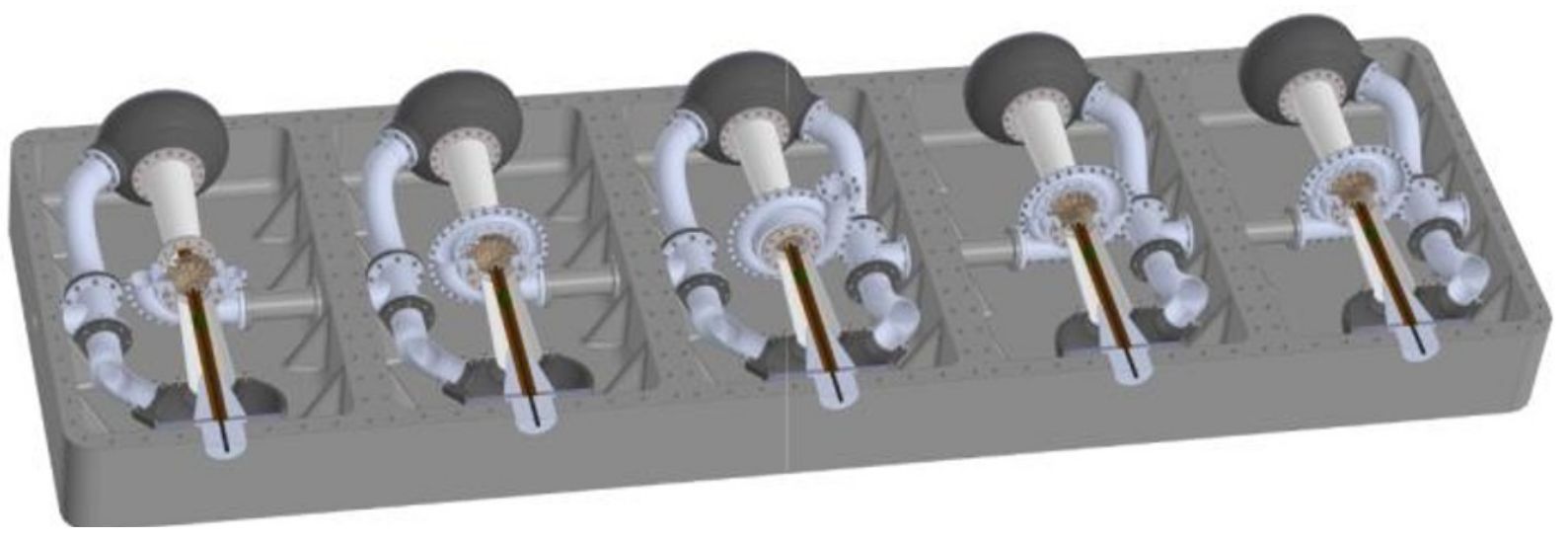

Fig. 4. Turbine unit.

To evaluate the required rotors diameter of generators, a preliminary strength calculation was carried out. According to which, the qualitative dependence of the power output from the shaft diameter was obtained. At the first stages, the corrosion-resistant steel 12X13, three shaft speeds and an admissible value of stresses in terms of rupture life equal to 100,000 hours under normal operating conditions were considered. In this case, it was taken into account that normal stresses rise with the diameter increase (3), but tangential stresses increase when decline of the diameter (4).

$$
\begin{gathered}
\sigma=\frac{1}{3} \cdot \rho \cdot n^{2} \cdot(0,5 \cdot D)^{2} \\
\tau=\frac{P \cdot 60}{2 \cdot \pi \cdot n \cdot W_{\rho}}
\end{gathered}
$$

where $\sigma$ is the normal stresses arising under the action of centrifugal forces $(\mathrm{Pa}), \tau$ is the shear stresses exerting a twisting effect on the shaft during the transfer of torque from the turbine unit to the generator $(\mathrm{Pa}), \rho$ is the density of the material $\left(\mathrm{kg} / \mathrm{m}^{3}\right), n$ is the rotational speed $(\mathrm{rev} / \mathrm{min}), D$ is shaft diameter $(\mathrm{m}), P$ is generated power $(\mathrm{W}), W_{\rho}$ is polar moment of resistance $\left(\mathrm{m}^{3}\right)$.

Thus, the dependence of the maximum output power from the shaft diameter at different rotational speeds was determined (Fig. 5).

As shown at the Fig. 5, with an increase in the generated power, the required cross-sectional area increases, which makes it possible to transmit the resulting torque. However, upon reaching a certain value of the diameter, the arising stresses from centrifugal forces begin to increase rapidly, which leads to a decrease in the possible transmitted power due to a decrease of the safety factors. Consequently, it is necessary either to reduce the speed and use fewer separate generators, or proceed to an increase in the generator sets, if high speeds of rotation are required due

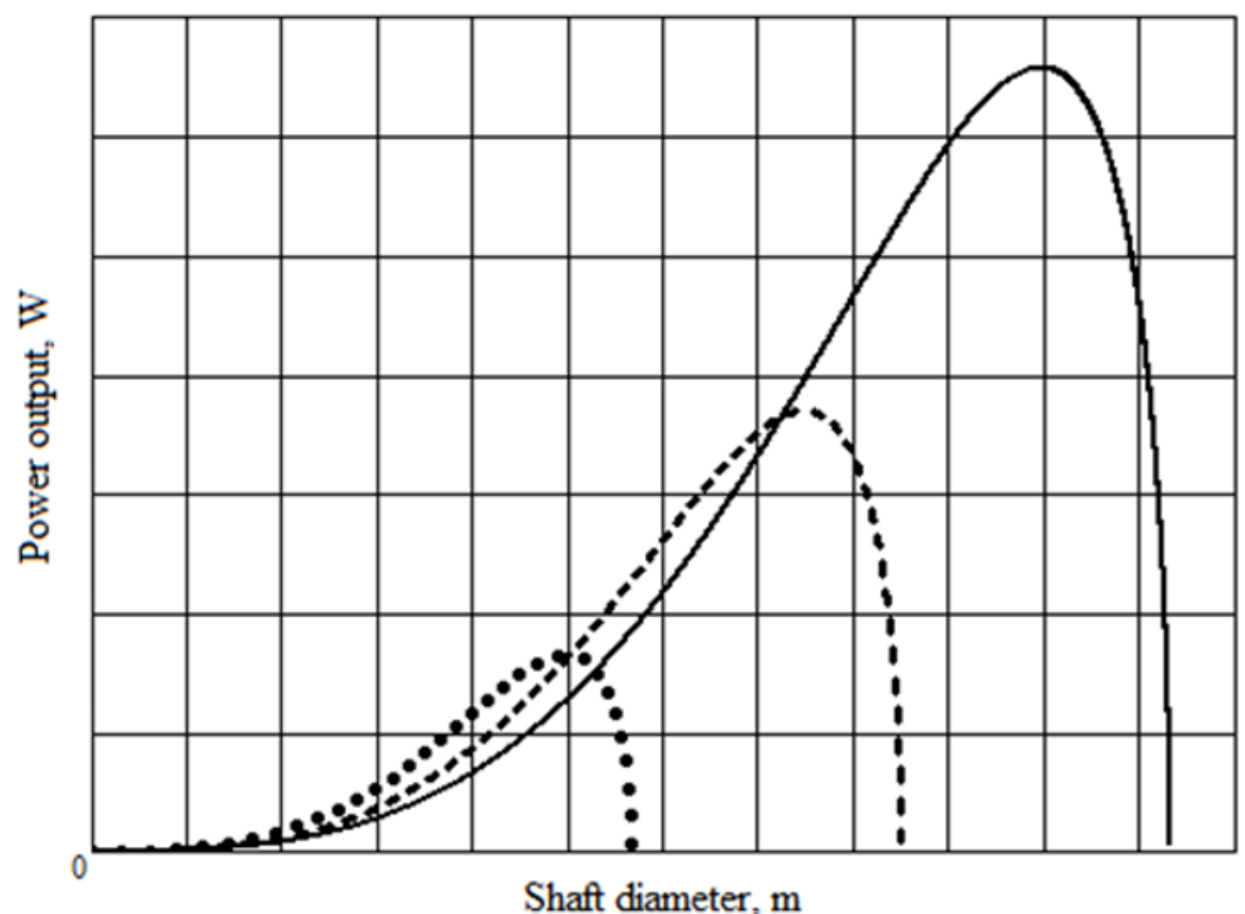

Fig. 5. Dependences of the generated power from the shaft diameter:

- is $18000 \mathrm{rpm},--$ is $24000 \mathrm{rpm}, \cdots$ is $36000 \mathrm{rpm}$. 
to the technological process.

As a result of the research, market analysis for auxiliary equipment and the draft elaboration of the main elements of CCCU, a 3D model of the station, operating according to the compressorless combined cycle was obtained, shown in Fig. 6.

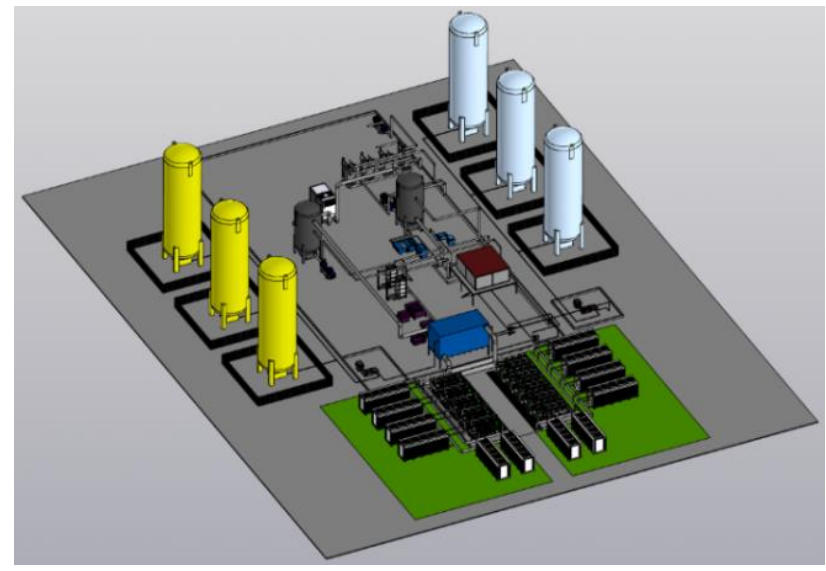

Fig. 6. 3D model of CCCU.

A description of compressorless combined cycle unit elements is presented in Fig. 7.

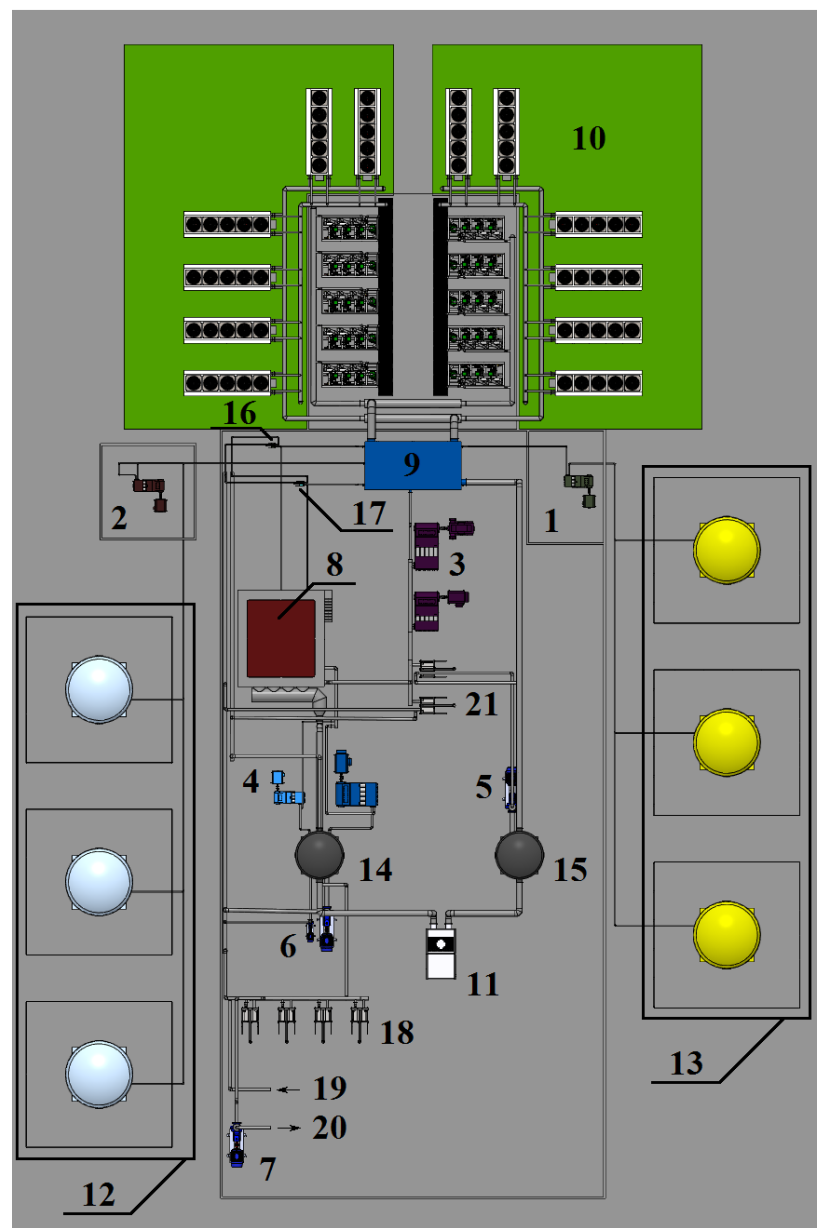

Fig. 7. Compressorless combined cycle unit schematic: 1, 2, 3, 4 - fuel, oxygen, carbon dioxide and water pumps, correspondingly; 5, 6 - circulation pumps; 7 network water pump; 8 - gas turbine unit; $9-\mathrm{CO}_{2}$ liquefaction device; 10 - refrigeration unit; 11 compressor of $\mathrm{CO}_{2} ; 12$ - cryogenic tanks with liquid $\mathrm{O}_{2}$; 13 - cryogenic tanks with liquid LNG (methane); 14 low-pressure contact condenser; 15 - high-pressure contact condenser; 16 - fuel heater; 17 - oxygen heater; 18 - heating system water heater; 19 - water withdrawal from heating system; 20 - supply of heated water to heating system.

The location of the tanks with liquid oxygen and methane must be located in isolated area from each other, so they were placed on opposite sides of the gas turbine unit.

The description of the thermodynamic cycle operation of CCCU can be conditionally begun from the moment when all the components of the working fluid are in the liquid phase. Feeding pumps for fuel 1, oxygen 2 , carbon dioxide 3 , and water 4 are used to increase their pressure. The fuel pump regulates the fuel supply. After the fuel pump, fuel is sequentially supplied to LNG cold recovery unit, installed in 9, and a fuel heater 16 , and then heated fuel enters a primary zone of a combustion chamber of GTU 8.

The oxygen supply is regulated by the oxygen pump 2. After the oxygen pump, oxygen first enters the cold recovery unit oxygen located in 9 , then into the oxygen heater 17 , after which the heated oxygen passes to the primary zone of the combustion chamber of GTU. Carbon dioxide pump 3 controls the supply of $\mathrm{CO}_{2}$. After the carbon dioxide pump, $\mathrm{CO}_{2}$ first enters the heater 21 , then it goes to the shell-and-tube recuperative heat exchanger (Fig. 3), located in the exhaust pipe of GTU. Heated $\mathrm{CO}_{2}$ is delivered simultaneously to the primary and secondary zones of the GTU combustion chamber, so as to ensure an acceptable quality of fuel combustion and the required temperature field at the exit from the combustion chamber.

A small fraction of $\mathrm{CO}_{2}$ is used to cool the hot parts in a flow path of the gas turbine unit 8 . The feedwater pump supplies water to a recuperative heat exchanger, and then, as well as $\mathrm{CO}_{2}, \mathrm{H}_{2} \mathrm{O}$ enters the primary and secondary zones of the combustion chamber. Thus, fuel is supplied to the combustion chamber in an amount that meets heat and electricity demand. The oxygen supply is regulated so that there is a minimum excess of oxygen, providing the required completeness of fuel combustion. The supply of $\mathrm{CO}_{2}$ maintains the set temperature of the working fluid in a turbine. The supply of $\mathrm{H}_{2} \mathrm{O}$ regulates the ratio of the generated heat and electrical energy.

The mixture of combustion products and ballasting components, obtained in the combustion chamber with a given temperature, represents the working fluid at the gas turbine inlet. In the steam-gas turbine, the working fluid expands, performing work. The work, performed in a steam-gas turbine, is converted into electricity by a generator. The waste working fluid is sent to recuperative heat exchangers, located at the outlet of the gas turbine unit, heating the ballasting components $\mathrm{H}_{2} \mathrm{O}$ and $\mathrm{CO}_{2}$. After the recuperative heat exchangers, the working fluid is directed to a low pressure contact condenser 14. At the outlet of the low pressure contact condenser, the working fluid is carbon dioxide with 
small impurities, including a small amount of $\mathrm{H}_{2} \mathrm{O}$. Therefore, in order to avoid freezing, the pressure of the working fluid is increased by the $\mathrm{CO}_{2}$ compressor 11 to 3.5 MPa. After that, the remains of $\mathrm{H}_{2} \mathrm{O}$ are condensed in a contact high-pressure condenser 15 . The working medium, cooled in the high-pressure contact condenser, is sent to the $\mathrm{CO}_{2}$ liquefaction device 9 . To liquefy $\mathrm{CO}_{2}$, the cold of liquid oxygen and fuel, coming from cryogenic tanks 12 and 13, respectively, are used. For this purpose, the $\mathrm{CO}_{2}$ liquefaction device includes the liquid oxygen and LNG cold utilizers.

The entire shortage of cold, required for $\mathrm{CO}_{2}$ liquefaction, is compensated by the refrigeration unit 10 . Each contact condenser has its own circulating water circuit. Circulation pumps 5 and 6 take water from the collectors of the contact condensers. In the circulation water circuit of the low-pressure condenser, after the circulation pump 6, water is divided into several streams. Most of the water is sent to the heating system water heater 18, after which it returns to the first section of the low pressure condenser. The rest of the water is supplied in parallel flows to the fuel heater 16, oxygen heater 17 and $\mathrm{CO}_{2}$ heater 21 .

The circulating water of the high pressure condenser 15, after the circulation pump 5, is supplied to the $\mathrm{CO}_{2}$ heater 21. After the heater, this water returns to the high pressure contact condenser 15 . The return network water 19 is supplied to heating water heater 18 , heats up to the temperature, required according to the temperature schedule, and returns to the heating system 20 by the heating water pump 7. The $\mathrm{CO}_{2}$ liquefaction device 9 is equipped with a system for removing noncondensable gases and bleeding-off an excess of liquid $\mathrm{CO}_{2}$. In addition, the selection of excess $\mathrm{H}_{2} \mathrm{O}$ is provided.

\section{Conclusion}

In the course of the research, the pilot model of the 60 MW installation was designed, operating according to the new promising compressorless combined cycle. The weight, size and functional parameters of the main parts of the energy complex were preliminarily determined. Auxiliary components of the compressorless combined cycle unit were selected, solutions for two groups of pumping equipment were found. Within the framework of the draft elaboration, preliminary weight, size and functional parameters of the turbine unit, plate and shelland-tube heat exchangers for returning the heat of combustion products have been determined. The obtained results make it possible to simulate the behavior (develop dynamic models) of the compressorless combined cycle unit in all operating modes (from startup to shutdown) under various conditions, as well as to carry out the feasibility study for the creation of such plants.

The research was financially supported by the Russian Science Foundation (project № 19-19-00558).

\section{References}

1. A.S. Kosoi, Y.A. Zeigarnik et al., Ther. Eng., 65, 9 (2018)

2. M.V. Sinkevich, Yu.A. Borisov, A.A. Kosoy, E.R. Ramazanov and O.S. Popel, AIP Conf. Proc. 2141, 030066 (2019)

3. M. Sinkevich, A. Kosoy, O. Popel, E3S Web of Conf., 209, 03023 (2020)

4. M. Sinkevich, Y. Borisov, A. Kosoy, E. Ramazanov, E3S Web of Conf., 209, 03022 (2020)

5. V.V. Datsenko, Y.A. Zeigarnik et al., Thermophysics and Aeromechanics. 27, 5 (2020)

6. E.R. Ramazanov, A.A. Kosoy, H. Khalife, J. of Ph.: Conf. Ser., 1675, 012094 (2020) 